\title{
Comparing infective complications from transrectal ultrasound guided prostate biopsy following transition to single dose oral ciprofloxacin prophylaxis
}

\author{
Matthew Farag ${ }^{1, *}$, Sophie Riddell ${ }^{1, *}$, John Daffy ${ }^{2}$, Lih-Ming Wong ${ }^{1,3}$ \\ Departments of ${ }^{1}$ Urology, ${ }^{2}$ Infectious Diseases, St Vincent's Hospital Melbourne, Victoria, ${ }^{3}$ Department of Surgery, University of Melbourne, Victoria, Australia
}

Purpose: To examine the incidence of infective complications post Transrectal Ultrasound Guided Prostate Biopsy (TRUSPB), after transition to preoperative administration of single dose oral ciprofloxacin.

Materials and Methods: A retrospective study of 766 consecutive patients undergoing TRUSPB at St Vincent's Hospital Melbourne (2002-2016). Antibiotic prophylaxis between 2002-2014 consisted of 3 days of perioperative oral norfloxacin \pm intravenous (IV) antibiotics (Group A, $n=687$ ). From November 2014 patients received a single dose of oral $750 \mathrm{mg}$ ciprofloxacin pre-biopsy (Group B, $n=79$ ), to align with the American Urological Association (AUA) and a Cochrane Database Systematic Review on Antibiotic Prophylaxis for TRUSPB. Groups were compared for all postoperative complications requiring representation and/or readmission within 30 days of biopsy.

Results: In Group A, 10 of 687 patients (1.5\%) re-presented with post-procedural fever (temperature $>38^{\circ} \mathrm{C}$ ), requiring readmission and IV antibiotic treatment, compared to 4 of the 79 patients $(5.1 \%)$ in Group B ( $p=0.02)$. Positive blood cultures were isolated in $0.9 \%(n=6$, Group A) versus 3.8\% ( $n=3$, Group B) $(p=0.02)$. The 4 infectious readmissions in Group B had no prior genitourinary infections, no recent travel and all had a Charlson Comorbidity Index scores $<2$. Two patients in Group B cultured Escherichia coli sensitive to ciprofloxacin despite receiving preoperative ciprofloxacin.

Conclusions: Antibiotic prophylaxis using single dose ciprofloxacin is associated with higher infective complications post TRUSPB. The episodes of ciprofloxacin sensitive E. coli bacteraemia in Group B suggest consideration of a longer course of perioperative antibiotic prophylaxis.

Keywords: Antibiotic prophylaxis; Biopsy; Ciprofloxacin; Fluoroquinolones; Prostate

This is an Open Access article distributed under the terms of the Creative Commons Attribution Non-Commercial License (http://creativecommons.org/licenses/by-nc/4.0) which permits unrestricted non-commercial use, distribution, and reproduction in any medium, provided the original work is properly cited.

Received: 14 September, 2018 - Accepted: 29 October, 2018

Corresponding Author: Matthew Farag

Department of Urology, St Vincent's Hospital Melbourne, 41 Victoria Parade, Fitzroy, Victoria 3065, Australia

TEL: +61-401537395, FAX: +61-392314737, E-mail: mattyfarag@hotmail.com

ORCID: https://orcid.org/0000-0003-3306-1531

*These authors contributed equally to this study and should be considered co-first authors. 


\section{INTRODUCTION}

Antibiotic prophylaxis for patients undergoing Transrectal Ultrasound Guided Prostate Biopsy (TRUSPB) remains controversial. Given that 18,263 prostate biopsies were completed in Australia in the last financial year, it is important to appropriately address this topic. Whilst infection rates following TRUSPB are traditionally low, estimated between $1 \%$ to $4 \%$ [1-6], when infection occurs it can be severe and even life threatening. A number of risk factors for infection have been established such as prior infections, overseas travel and a Charlson Comorbidity Index (CCI) score greater than two [5,7-10].

In recent times, guidelines regarding the best practice antibiotic prophylaxis prior to TRUSPB have changed. In 2011 the Cochrane Database of Systematic Reviews released a publication on Antibiotic Prophylaxis for Transrectal Prostate Biopsy. It outlined the significant benefit of prophylactic antibiotics for TRUSPB, with the most evidence for fluoroquinolones [4]. This reflected the American Urological Association's (AUA) best practice policy statement from 2008, which now provides the most current clinical recommendation on antimicrobial prophylaxis for TRUSPB. It recommends fluoroquinolones or 1st/2nd/3rd generation cephalosporins for $\leq 24$ hours (level of evidence 1b) [11]. The lack of benefit seen from longer antibiotic regimes and the potential for pharmacological side effects from an increased duration of treatment formed the basis of this recommendation. As a result, many hospitals around the world transitioned to a single dose antibiotic regime [12-14].

To align with the AUA guideline and the Cochrane Review our institution adopted a preoperative regime of administering $750 \mathrm{mg}$ single dose oral ciprofloxacin prior to TRUSPB, as of November 2014.

However, due to growing concerns within our institution that a single dose of ciprofloxacin was not an adequate duration of antibiotic prophylaxis, this study was proposed. Our aim was to compare the incidence of infective complications before and after the transition, and to evaluate the antibiotic sensitivities of bacterial pathogens in patients with positive cultures post TRUSPB.

\section{MATERIALS AND METHODS}

\section{Patient selection}

A retrospective study of patients undergoing TRUSPB at a single tertiary referral institution; St Vincent's Hospital Melbourne, Australia, was undertaken. Patients were identified from surgical procedure codes, between 2002 and
2016. Readmissions within 30 days were found by manually reviewing the 'hospital attendance' component of patient online records. Approval was gained from the St Vincent's Hospital Melbourne ethics committee.

Patient characteristics included age at biopsy and prebiopsy prostate specific antigen (PSA). Charlson Comorbidity Index (CCI) scores were collected from the hospital admission database to identify any predisposing comorbidities such as diabetes, cerebrovascular or cardiac disease. Age related mortality risk and CCI were used to calculate a combined score, which in turn provides an estimated 10-year survival (Table 1). No patients were excluded from the study.

\section{Method}

Patients undergoing TRUSPB between May 2002 and November 2014 were prescribed 3 days of perioperative fluoroquinolone (norfloxacin); prescriptions were provided at the pre-admission clinic with compliance confirmed on day of biopsy. Intraoperative, intravenous (IV) antibiotics such as $1 \mathrm{~g}$ ceftriaxone or $240 \mathrm{mg}$ gentamicin, were given at the discretion of the treating urologist (Group A). From November 2014 the antibiotic prophylaxis protocol was changed to a single dose of fluoroquinolone (oral ciprofloxacin $750 \mathrm{mg}$ ), administered by the surgical team, 1 hour before the patient's TRUSPB (Group B). All patients with additional risk factors for infection who received individualised antibiotic regimens throughout the study period were included in Group A as they were ineligible for single dose oral ciprofloxacin alone. All patients undertook a pre-biopsy urine microscopy, culture and sensitivity, and positive cultures were treated appropriately.

Surgeons performed TRUSPB techniques in a standard extended template. A double-layered transducer cover was placed over the ultrasound probe (BK medical US probe $8818 \mathrm{~K}$ or Mindray US probe model 65EC10EA). Biopsies were usually performed using 18G tru-cut needle (Bard 18 g core biopsy needle gun). Preoperative rectal swabs were not taken, and no enemas or iodine suppositories were administered.

The microbiological methods for collection, processing and culture of specimens remained similar throughout the study period. Blood cultures were processed using the BACTEC (BD Diagnostics, Sparks, MD, USA). Both blood and urine were subcultured onto routine bacteriology media with subsequent isolates being identified by Vitek-2 (bioMérieux, Marcy-l'Étoile, France) (2002-2012) or Matrix assisted laser desorption time of flight mass spectrometry (MALDITOF; Bruker Daltonics, Bremen, Germany) (2012-2016). Antimicrobial susceptibility testing was performed using 
Table 1. Patient and biopsy characteristics $(n=766)$

\begin{tabular}{|c|c|c|c|}
\hline Characteristic & Group A & Group B & $\mathrm{p}$-value \\
\hline Number of patients & $687(89.7)$ & $79(10.3)$ & \\
\hline Age (y) & $66.86 \pm 8.69$ & $65.89 \pm 8.05$ & 0.34 \\
\hline PSA at diagnosis (ng/mL) & $9.0(6.0-15.7)$ & $8.2(6.0-12.6)$ & 0.16 \\
\hline $\mathrm{CCl}$ & $0.0(0.0-2.0)$ & $0.0(0.0-2.0)$ & $0.05^{\mathrm{b}}$ \\
\hline Age related mortality risk & $2.0(2.0-3.0)$ & $2.0(2.0-3.0)$ & 0.30 \\
\hline Combined age-CCl score ${ }^{a}$ & $3.0(2.0-5.0)$ & $3.0(2.0-5.0)$ & 0.50 \\
\hline Estimated 10 year survival & $78.0(21.0-90.0)$ & $78.0(21.0-90.0)$ & 0.50 \\
\hline \multicolumn{4}{|l|}{ Biopsy characteristic } \\
\hline Total cores & $16.0(13.0-19.0)$ & $14.0(12.0-18.0)$ & 0.09 \\
\hline Patients with positive cores & $334(48.6)$ & $50(63.3)$ & \\
\hline Number of positive cores & $5.00(2.0-9.0)$ & $4.0(2.0-8.0)$ & 0.64 \\
\hline Highest Gleason score & $3.0(3.0-4.0)$ & $3.5(3.0-4.0)$ & 0.81 \\
\hline
\end{tabular}

Values are presented as number (\%), mean \pm standard deviation, or median (interquartile range).

PSA, prostate specific antigen; $\mathrm{CCl}$, Charlson Comorbidity Index.

${ }^{a}:$ Combined score=single prognostic variable combining age related mortality risk and $\mathrm{CCl}$, that is indicative of subsequent risk.

${ }^{\mathrm{b}}$ :The two sample Wilcoxon-Mann-Whitney test was used for non-normally distributed continuous and ordinal variables, this $p$-value approaches significance (0.05), few subjects in Group B had multiple comorbidities placing them outside of the interquartile range and increasing Group B's rank sum (without affecting median and interquartile range).

Vitek-2. Clinical and Laboratory Standards Institute (CLSI) interpretive criteria were applied [15]. The CLSI breakpoint for ciprofloxacin susceptibility for Enterobacteriaecea remained the same throughout the study period at $\leq 1 \mu \mathrm{g} / \mathrm{mL}$.

The primary outcome was readmission to our institution within 30 days following TRUSPB. Readmissions were further stratified into planned and unplanned, with unplanned then divided into febrile and non-febrile categories. Post-procedural fever was defined as temperature $>38^{\circ} \mathrm{C}$. Secondary outcomes were the incidence of bacteraemia (positive blood culture) and urinary tract infection (UTI), i.e,, a positive urine culture with fever, dysuria and/or polyuria. Antibiotic sensitivities for positive urine and blood cultures for patients readmitted with fever were recorded.

\section{Statistical analysis}

Calculations were performed using Stata/MP version 13.0 for Mac (Stata Co, College Station, TX, USA). Variables were checked for skewness and kurtosis to determine normality. Clinical and demographic features are presented as medians [interquartile range] and means ( \pm standard deviation) for non-parametric and parametric data respectively. Differences between continuous parametric variables were examined with the t-test; the Wilcoxon rank-sum test or the WilcoxonMann-Whitney test were used for non-normally distributed continuous and ordinal variables, while differences between dichotomous variables were evaluated with the $\chi^{2}$ test or the Fishers exact test (Tables 1, 2). A p-values throughout the results were two sided.

\section{RESULTS}

A total of 766 consecutive patients underwent TRUSPB. Group A included 687 patients (89.7\%) and Group B, 79 patients (10.3\%). Patients in both groups were statistically alike regarding demographic characteristics (Table 1). The mean age of patients was $66.86 \pm 8.69$ and $65.89 \pm 8.05$ in Groups A and B, respectively $(p=0.34)$. TRUSPB characteristics including PSA prior to biopsy, total cores, number of positive cores and highest Gleason score were also statistically similar (Table 1). There was no difference in combined ageCCI score or estimated 10-year survival between the groups $(\mathrm{p}=0.50)$ (Table 1).

Comparison of readmissions within 30 days post TRUSPB is shown in Table 2. There was no statistical difference in total 30 day unplanned readmissions between Group A ( $n=38$, $5.5 \%)$ and Group B ( $n=4,5.1 \%)$. The incidence of non-febrile unplanned readmission including hematuria, per rectal bleeding and urinary retention was not significantly different between the two groups (Table 2).

Febrile readmissions occurred significantly more frequently in patients receiving single dose oral ciprofloxacin (Group $\mathrm{A}=1.5 \%$, Group $\mathrm{B}=5.1 \%, \mathrm{p}=0.02$ ). Febrile readmissions yielding a positive blood culture were significantly higher in Group B (3.8\%) than in Group A (0.9\%) $(\mathrm{p}=0.02)$. Febrile readmissions yielding a positive urine culture were also significantly higher in Group B (3.8\%) than in Group A (0.7\%) $(p=0.02)$ (Table 2). There was no difference between lengths of stay for post-procedure febrile readmissions between the 
Table 2. Readmissions within 30 days post TRUSPB

\begin{tabular}{|c|c|c|c|c|}
\hline Readmission type & Group A ( $n=687)$ & Group B ( $n=79)$ & Total $(n=766)$ & p-value \\
\hline Total readmissions $<30$ days & $54(7.9)$ & $4(5.1)$ & $58(7.6)$ & 0.37 \\
\hline Planned & $14(2.0)$ & $0(0.0)$ & $14(1.8)$ & 0.20 \\
\hline Unplanned & $38(5.5)$ & $4(5.1)$ & $42(5.5)$ & 0.86 \\
\hline Unknown & $2(0.3)$ & $0(0.0)$ & $2(0.3)$ & 0.63 \\
\hline Unplanned readmissions & $38(5.5)$ & $4(5.1)$ & $42(5.5)$ & 0.86 \\
\hline Febrile readmissions & $10(1.5)$ & $4(5.1)$ & $14(1.8)$ & 0.02 \\
\hline ICU admission & 1 & 0 & 1 & 1.00 \\
\hline Length of stay (d) & $8.20 \pm 4.26$ & $6.00 \pm 4.08$ & NA & 0.40 \\
\hline Day of representation postoperative & $4.30 \pm 3.89$ & $2.50 \pm 1.00$ & NA & 0.39 \\
\hline Positive urine culture & $5(0.7)$ & $3(3.8)$ & $8(1.0)$ & 0.01 \\
\hline Urine cultures sensitive to ciprofloxacin & 2 & 2 & 4 & 0.06 \\
\hline Urine cultures resistant to ciprofloxacin & 3 & 1 & 4 & 0.35 \\
\hline Positive blood culture & $6(0.9)$ & $3(3.8)$ & $9(1.2)$ & 0.02 \\
\hline Blood cultures sensitive to ciprofloxacin & 3 & 2 & 5 & 0.09 \\
\hline Blood cultures resistant to ciprofloxacin & 3 & 1 & 4 & 0.35 \\
\hline Non-febrile readmissions & $28(4.1)$ & $0(0.0)$ & $28(3.7)$ & 0.10 \\
\hline Hematuria & 9 & 0 & 9 & 0.61 \\
\hline Per rectal bleeding & 2 & 0 & 2 & 1.00 \\
\hline Urinary retention & 1 & 0 & 1 & 1.00 \\
\hline Other/unrelated & 16 & 0 & 16 & 0.40 \\
\hline
\end{tabular}

Values are presented as number (\%), number only, or mean \pm standard deviation.

TRUSPB, Transrectal Ultrasound Guided Prostate Biopsy; ICU, intensive care unit; NA, not applicable.

Table 3. Febrile readmissions; comparison of antibiotic administration to bacterial sensitivity

\begin{tabular}{|c|c|c|c|c|c|c|c|}
\hline Microbiology & No. & Oral $\mathrm{FQ}^{\mathrm{a}}$ given & $\begin{array}{c}\text { Sensitive to } \\
\text { FQ }^{\mathrm{a}}\end{array}$ & $\begin{array}{l}\text { IV ceft or } \\
\text { gent given }\end{array}$ & $\begin{array}{l}\text { Sensitive to } \\
\text { ceft or gent }\end{array}$ & $\begin{array}{l}\text { Resistant to } \\
\text { all Abx given }\end{array}$ & $\begin{array}{l}\text { Sensitive to } \\
\text { all Abx given }\end{array}$ \\
\hline \multicolumn{8}{|l|}{ Group A $(n=10)$} \\
\hline Positive BC only & $3^{b}$ & 3 & 3 & 2 & 2 & 0 & 3 \\
\hline Positive UC only & 2 & 2 & 2 & 2 & 2 & 0 & 2 \\
\hline Positive $\mathrm{BC}+\mathrm{UC}$ & 3 & 3 & 0 & 0 & 3 & 3 & 0 \\
\hline Negative cultures & 2 & 2 & - & 2 & - & - & - \\
\hline \multicolumn{8}{|l|}{ Group B $(n=4)$} \\
\hline Positive BC only & 0 & 0 & 0 & 0 & 0 & 0 & 0 \\
\hline Positive UC only & 0 & 0 & 0 & 0 & 0 & 0 & 0 \\
\hline Positive $\mathrm{BC}+\mathrm{UC}$ & 3 & 3 & 2 & 0 & 3 & 1 & 2 \\
\hline Negative cultures & 1 & 1 & - & 0 & - & - & - \\
\hline
\end{tabular}

FQ, fluoroquinolone; IV, intravenous; ceft, ceftriaxone; gent, gentamycin; Abx, antibiotics; BC, blood cultures; UC, urine cultures.

a:FQ given in Group A=oral norfloxacin, 400 mg, twice a day, for 3 days perioperatively. Group B=oral ciprofloxacin, 750 mg, 1 hour preoperatively.

${ }^{\mathrm{b}}$ :One patient from this group was non-compliant with preoperative oral antibiotics. One patient in this group cultured Streptococcus constellatus and Bacteroides fragilis, all other bacteria were Escherichia coli.

groups. Overall, only one patient required intensive care unit (ICU) admission and there was no mortality in our cohort.

Across all febrile readmissions, no patients had documented travel history within 12 months pre-TRUSPB. The four patients in Group B who were readmitted with fever were further evaluated for potential risk factors (Supplementary Table 1) [5,7-10]. All four patients had a CCI less than 2. One patient who grew ciprofloxacin-sensitive
Escherichia coli was on aspirin and had undergone an uncomplicated TRUSPB one year prior. No identifiable risk factors were found in the remaining 3 patients.

Comparison between antibiotic administration and bacterial sensitivity in all febrile patients is shown in Table 3. E. coli was responsible for all readmissions with bacteraemia and/or bacteriuria except one patient in Group A, who grew Streptococcus constellatus and Bacteroides fragilis in blood 
only. In Group B, 2 of the 3 patients readmitted with fever subsequently cultured organisms from blood and urine sensitive to ciprofloxacin despite preoperative single dose oral ciprofloxacin. Three patients in Group A (0.4\%) and one patient in Group B (1.3\%) cultured bacteria resistant to fluoroquinolones. A detailed summary of all febrile readmissions is presented in Supplementary Table 2.

\section{DISCUSSION}

In our institution, the incidence of post-procedural fever following TRUSPB significantly increased following transition to the single dose oral fluoroquinolone regime (1.5\% vs. $5.1 \%$, p=0.02). Results from The Department of Health's 'Victorian Admitted Episodes Dataset', showed that between 2007 to 2012, state-wide readmissions resulting from infection, within seven days of biopsy, was $1.7 \%$ [1]. Thus, prior to the transition to single dose antibiotic prophylaxis, our institution had comparative infection rates to local standards (1.5\%), even with a wider 30-day (cf. 7-day) readmission window.

In the literature, post TRUSPB infection rates range from $1 \%$ to $4 \%[5,6]$. Variation is attributable to differences in antibiotic prophylaxis regimens, local bacterial antibiotic sensitivities, cohort exclusion criteria and methods of reporting such as 7 versus 30-day windows. Incidence of infective complications post TRUSPB using single dose ciprofloxacin prophylaxis is limited. Lundström et al. [10] completed a large $(n=51,321)$ national Swedish study reviewing patients with prostate cancer undergoing TRUSPB. Of these patients, $6 \%$ were prescribed antibiotics within 30 days, but only $1 \%$ of patients were hospitalised for infection. A national Swedish audit at the time stated $83 \%$ of antibiotic prophylaxis used was ciprofloxacin and $64 \%$ was a single dose of oral antibiotics immediately before biopsy [10]. In another study by Lindstedt et al. [9], they reported one of the lowest rates of complications in a cohort receiving single dose ciprofloxacin prophylaxis, with $0.9 \%$ for UTI and $0.3 \%$ for hospital admission post TRUSPB. However, this study looked at healthy subjects only, excluding $16 \%$ of their initial cohort due to identifiable risk factors for infection. Eight of the 12 patients readmitted with infection were then found to have infective risk factors on further analysis. Hence, these low infection rates following single dose ciprofloxacin prophylaxis are not generalisable to an average population such as our study cohort.

Our institution's antibiotic prophylaxis regime was altered to follow the AUA best practice policy statement 2008 and the Cochrane Systematic Review from 2011. The
Cochrane Systematic Review supports the use of antibiotic prophylaxis, in particular fluoroquinolones [4,13]. In the systematic review no individual randomised controlled trial (RCT) showed a statistically significant benefit of single dose over multi-dose treatment for reducing fever, UTI or bacteraemia. A forest plot comparison within the systematic review incorporating four RCT's, supported multi-dose as opposed to single dose antibiotic prophylaxis in reducing bacteriuria (relative risk, 1.98; 95\% confidence interval, 1.18; 3.33) [4]. Furthermore, forest plot comparisons all favoured multi over single dose antibiotic prophylaxis for reducing fever, UTI and hospitalisation [4]. The trend from the Cochrane Systematic Review is that multi dose antibiotic prophylaxis is favoured, however, large scale RCTs would be required to further evaluate an appropriate duration.

The AUA policy advising < 24 hours of antibiotic prophylaxis is based on three studies, the largest of which had 363 participants. In the RCT $(\mathrm{n}=231)$ by Aron et al. [12], they compared placebo to ciprofloxacin (500 mg) and tinidazole $(600 \mathrm{mg}$ ) either as a single dose or as a three day bi-daily course. Infective complications in the three day perioperative antibiotic group (10.3\%) offered no statistically significant benefit over single dose (7.5\%) [12]. Shigemura et al. [3], in a non-randomised prospective study $(n=236)$ compared one day of levofloxacin $600 \mathrm{mg}$ to a three-day $300 \mathrm{mg}$ regimen and found infective complications were equivalent in each group, $1.6 \%$ and $1.79 \%$, respectively. The one-day regimen included two postoperative doses at two and eight hours [3]. Finally, Sabbagh et al. [2] also compared a one-day versus three-day antibiotic regimen in a randomised open label trial $(n=363)$. Seven days post biopsy telephone follow up recorded levels of infection at only $0.55 \%$ for both groups. In all three trials, the lack of benefit seen from the longer antibiotic regimens formed the basis of the recommendation of $<24$ hours of antibiotic prophylaxis.

Our results support longer course antibiotic prophylaxis. For patients receiving single dose ciprofloxacin, two of the four patients that were re-admitted with fever cultured organisms sensitive to ciprofloxacin. Neither patient had had previous genitourinary infections or recent travel and both had a CCI of less than two. This suggests a longer course of ciprofloxacin would have been required to prevent these infections, and accounts for the significantly increased infection rate in Group B compared to Group A.

Three of the patients in Group A (0.4\%) and one of the patients in Group B (1.3\%) cultured bacteria that was resistant to all the antibiotics they received. In changing our antibiotic prophylaxis policy, consideration was made for the resistant organisms found in Australia due to our 
proximity to South East Asia. A recent review of antibiotic resistance in Australia has indicated ciprofloxacin resistance in $6.2 \%-8.7 \%$ of $E$. coli isolates including both community and hospital settings [16]. As antibiotic resistance increases, a trans perineal approach should be considered in high-risk patients.

The limitations of our study include the retrospective nature and lack of randomisation. The separation of Groups A and B with respect to time may have influenced results due to changing local bacterial profiles. We did not retrieve missing data from pre electronic records for risk factors for infection, nor the exact antibiotics given for patients in Group A. The low number of biopsies per year of approximately fifty may be a possible source of bias. There may be a cohort of patients who did not re-present to our institution for their biopsy complications and hence have not been captured.

\section{CONCLUSIONS}

Consensus regarding the most effective course of prophylaxis for TRUSPB has not yet been reached. Further investigation with prospective trials is required, ideally comparing 24 hours to 3 days of oral antibiotic prophylaxis [17]. Extended duration of therapy to reduce infective complications must be weighed against pharmacological side effects and risk of resistant organism propagation. Our study suggests that single dose ciprofloxacin is inadequate prophylaxis for TRUSPB. This finding requires a targeted prospective trial, accounting for the increasing antibiotic resistance that is now widely acknowledged in the medical field. A multi-dose antibiotic regime has since been instituted at our hospital in consultation with our infectious diseases department.

\section{CONFLICTS OF INTEREST}

The authors have nothing to disclose.

\section{ACKNOWLEDGMENTS}

\author{
St Vincent's Pathology Laboratory, St Vincent's Hospital \\ Melbourne, Australia.
}

\section{SUPPLEMENTARY MATERIALS}

Scan this QR code to see the supplementary materials, or visit https://www.icurology.org/src/sm/icurology-60-54-s001.pdf.

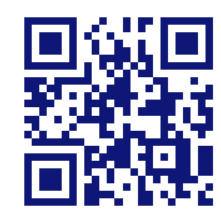

\section{REFERENCES}

1. Roth H, Millar JL, Cheng AC, Byrne A, Evans S, Grummet J. The state of TRUS biopsy sepsis: readmissions to Victorian hospitals with TRUS biopsy-related infection over 5 years. BJU Int 2015;116 Suppl 3:49-53.

2. Sabbagh R, McCormack M, Péloquin F, Faucher R, Perreault JP, Perrotte $\mathrm{P}$, et al. A prospective randomized trial of 1-day versus 3-day antibiotic prophylaxis for transrectal ultrasound guided prostate biopsy. Can J Urol 2004;11:2216-9.

3. Shigemura K, Tanaka K, Yasuda M, Ishihara S, Muratani T, Deguchi T, et al. Efficacy of 1-day prophylaxis medication with fluoroquinolone for prostate biopsy. World J Urol 2005;23:35660.

4. Zani EL, Clark OA, Rodrigues Netto N Jr. Antibiotic prophylaxis for transrectal prostate biopsy. Cochrane Database Syst Rev 2011;(5):CD006576.

5. Loeb S, Vellekoop A, Ahmed HU, Catto J, Emberton M, Nam $\mathrm{R}$, et al. Systematic review of complications of prostate biopsy. Eur Urol 2013;64:876-92.

6. Wagenlehner FM, van Oostrum E, Tenke P, Tandogdu Z, Çek $\mathrm{M}$, Grabe M, et al. Infective complications after prostate biopsy: outcome of the Global Prevalence Study of Infections in Urology (GPIU) 2010 and 2011, a prospective multinational multicentre prostate biopsy study. Eur Urol 2013;63:521-7.

7. Aus G, Ahlgren G, Bergdahl S, Hugosson J. Infection after transrectal core biopsies of the prostate--risk factors and antibiotic prophylaxis. Br J Urol 1996;77:851-5.

8. Bruyère F, Malavaud S, Bertrand P, Decock A, Cariou G, Doublet JD, et al. Prosbiotate: a multicenter, prospective analysis of infectious complications after prostate biopsy. J Urol 2015;193:145-50.

9. Lindstedt S, Lindström U, Ljunggren E, Wullt B, Grabe M. Single-dose antibiotic prophylaxis in core prostate biopsy: impact of timing and identification of risk factors. Eur Urol 2006;50:832-7.

10. Lundström KJ, Drevin L, Carlsson S, Garmo H, Loeb S, Stattin $\mathrm{P}$, et al. Nationwide population based study of infections after transrectal ultrasound guided prostate biopsy. J Urol 2014;192:1116-22.

11. Wolf JS Jr, Bennett CJ, Dmochowski RR, Hollenbeck BK, Pearle MS, Schaeffer AJ; Urologic Surgery Antimicrobial Prophylaxis Best Practice Policy Panel. Best practice policy state- 
ment on urologic surgery antimicrobial prophylaxis. J Urol 2008;179:1379-90.

12. Aron M, Rajeev TP, Gupta NP. Antibiotic prophylaxis for transrectal needle biopsy of the prostate: a randomized controlled study. BJU Int 2000;85:682-5.

13. Kapoor DA, Klimberg IW, Malek GH, Wegenke JD, Cox CE, Patterson AL, et al. Single-dose oral ciprofloxacin versus placebo for prophylaxis during transrectal prostate biopsy. Urology 1998;52:552-8.

14. Antibiotic Expert Groups [Internet]. Therapeutic guidelines; 2014 Nov [cited 2016 Feb 8]. Available from: https://tgldcdptg-org-au.ezp.lib.unimelb.edu.au/viewTopic?topicfile=surgicalprophylaxis\#toc_d1e1921.

15. Patel JB; Clinical and Laboratory Standards Institute. Per- formance standards for antimicrobial susceptibility testing: twenty-fifth informational supplement. Wayne: Clinical and Laboratory Standards Institute; 2015.

16. Australian Commission on Safety and Quality in Health Care (ACSQHC). AURA 2016: first Australian report on antimicrobial use and resistance in human health. Sydney: Australian Commission on Safety and Quality in Health Care (ACSQHC); 2016.

17. Which strategies are effective for reducing the risk of infective complications in men undergoing prostate biopsy? [Internet]. National Institute for Health Research; 2015 Sep 21 [cited 2016 Feb 12]. Available from: http://www.crd.york.ac.uk/PROSPERO/display_record.php?ID=CRD42015026354. 
Farag et al.

Investig Clin Urol 2019;60:54-60. January 2019. https://doi.org/10.4111/icu.2019.60.1.54

Supplementary Table 1. Risk factors for febrile readmissions in Group B

\begin{tabular}{|c|c|c|c|c|}
\hline Risk factor & Patient 1 & Patient 2 & Patient 3 & Patient 4 \\
\hline $\mathrm{CCl}>2$ & $\begin{array}{l}\text { No- } 2(77.5 \% \text { estimated } \\
10 \text { y survival) }\end{array}$ & $\begin{array}{l}\text { No- } 0 \text { ( } 90.1 \% \text { estimated } \\
10 \text { y survival) }\end{array}$ & $\begin{array}{l}\text { No- } 2 \text { ( } 53.4 \% \text { estimated } \\
10 \text { y survival) }\end{array}$ & $\begin{array}{l}\text { No- } 2 \text { ( } 21.4 \% \text { estimated } \\
10 \text { y survival) }\end{array}$ \\
\hline Prior infection (6 mo preceding biopsy) & No & No & No & No \\
\hline Diabetes & No & No & No & No \\
\hline Pharmacological treatment of LUTS/BPH & No & No & No & No \\
\hline Antiplatelets & No & No & Yes (aspirin) & No \\
\hline Increased number of cores $>7$ & Yes (21) & Yes (14) & Yes (13) & Yes (14) \\
\hline Positive urine MSU/dipstick preoperative & No & No & No & No \\
\hline IDC & No & No & No & No \\
\hline Prostatitis & No & No & No & No \\
\hline Steroids & No & No & No & No \\
\hline $\begin{array}{l}\text { Travelled overseas ( } 12 \text { mo preceding } \\
\text { biopsy) }\end{array}$ & No & No & No & No \\
\hline Used antibiotics (4 wk preceding biopsy) & No & No & No & No \\
\hline $\begin{array}{l}\text { Non-compliance with preoperative } \\
\text { Antibiotics }\end{array}$ & No & No & No & No \\
\hline Previous TRUSPB & No & No & $\begin{array}{l}\text { Yes- } 1 \text { previous TRUSPB } \\
1 \text { y preceding this } \\
\text { TRUSPB }\end{array}$ & No \\
\hline
\end{tabular}

$\mathrm{CCl}$, Charlson Comorbidity Index; LUTS, lower urinary tract symptoms; BPH, benign prostatic hypertrophy; MSU, mid-stream urine; IDC, indwelling catheter; TRUSPB, Transrectal Ultrasound Guided Prostate Biopsy. 


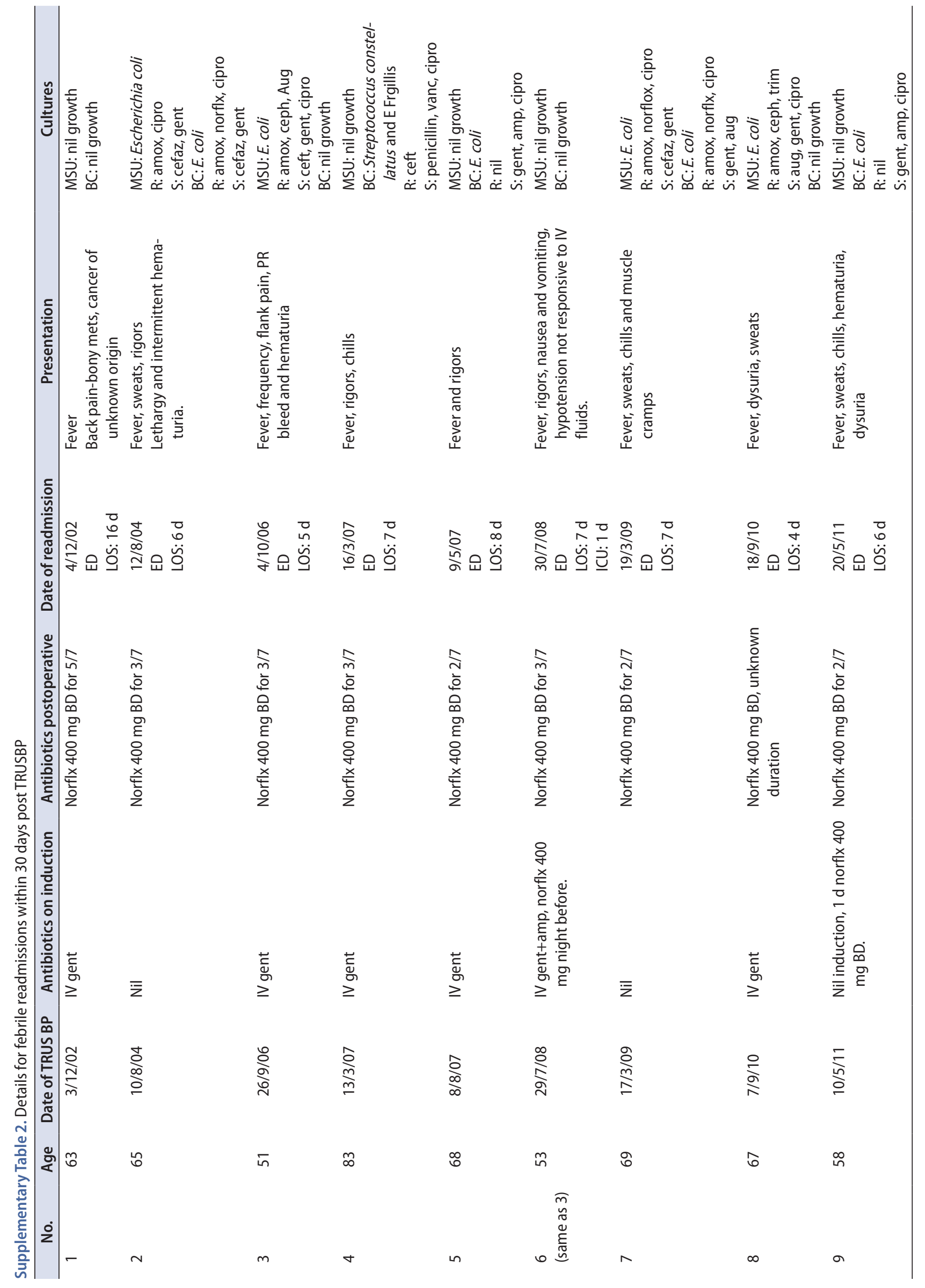




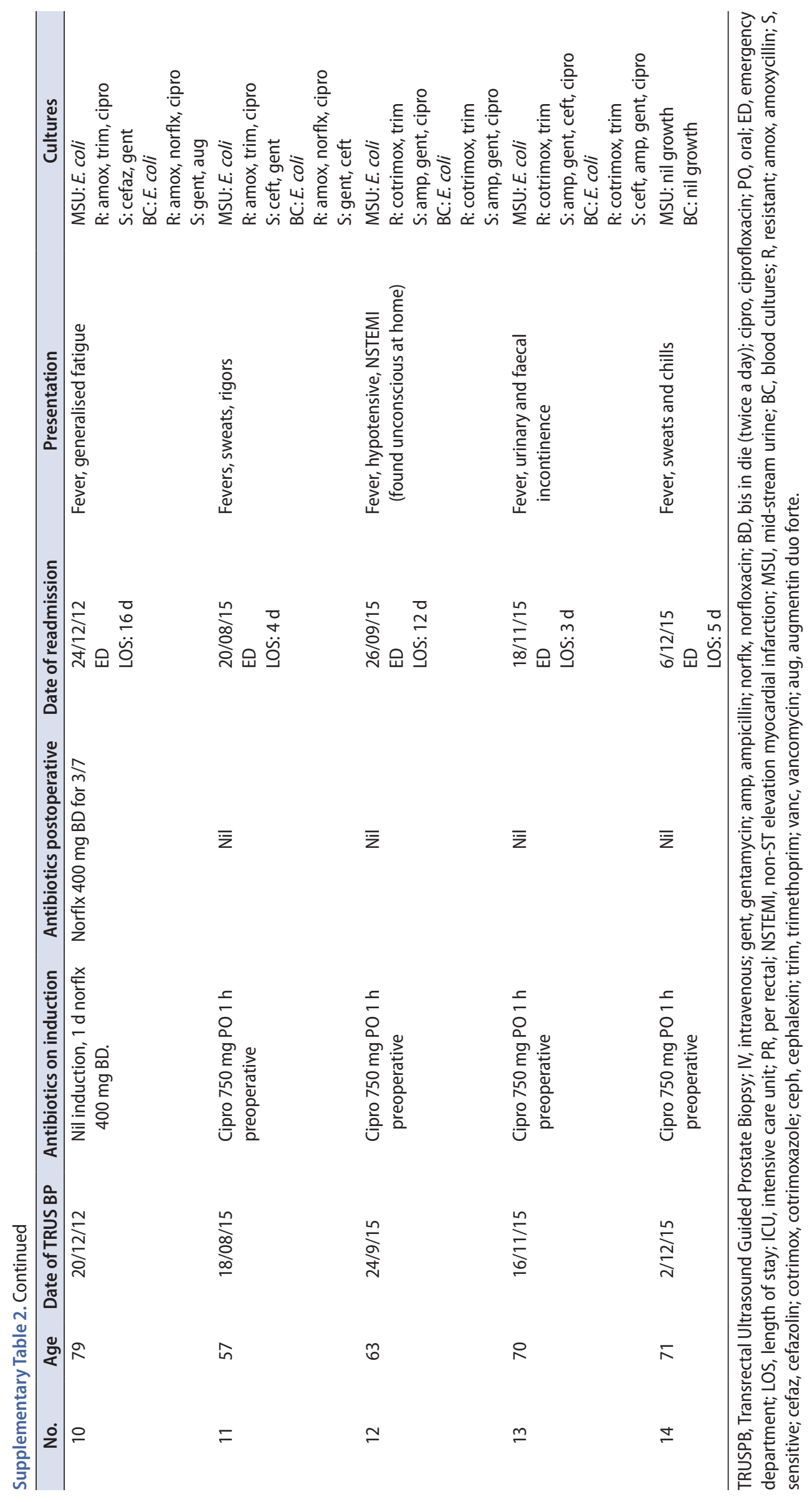

